MODELING, IDENTIFICATION AND CONTROL, 1983, VOL. 4, NO. 2, 107-116

doi:10.4173/mic.1983.2.5

\title{
Optimal load allocation
}

\section{Ø. ANDREASSEN $\dagger$ and T. O. OLSEN $\ddagger$}

Keywords: Boiler load allocation, optimization, sub-optimal solutions, convergence.

An algorithm for optimal boiler load allocation is compared with a sub-optimal algorithm and with equal load distribution. Advantages and disadvantages of the optimal and sub-optimal algorithms are discussed, and by a practical example it is demonstrated that the optimal algorithm may give substantial cost reductions.

\section{Introduction}

Optimal load allocation in steam generating plants has important energy conservation and cost minimization aspects. The load allocation problem may be treated as an optimization problem, with minimum generating costs as the objective. Due to the amount of computation which is required to solve the resulting non-linear minimization problem, several simpler allocation schemes have been proposed and used. This paper investigates the convergence of one particular sub-optimal method, and compares it to an optimal solution. Based on theoretical considerations and application examples, this paper advocates the use of an optimal allocation scheme.

\section{Problem statement}

The optimal load allocation consists of distributing the given total plant steam demand $L_{T}$ between $N$ boilers, allocating each boiler the steam $\operatorname{load} L_{i}(i=1, \ldots, N)$, so that the total steam generation cost

$$
C=\sum_{i=1}^{N} C_{i}
$$

is minimized subject to

$$
\begin{aligned}
& \sum_{i=1}^{N} L_{i}=L_{T} \text { (total steam demand must be satisfied) } \\
& L_{i_{\min }} \leqslant L_{i} \leqslant L_{i_{\max }} \text { or } L_{i}=0 \text { (boiler capacity limits) }
\end{aligned}
$$

Solving eqn. (2) for say $L_{N}$, the resulting allocation problem is a $N-1$ dimensional optimization problem.

As in Leffler and Shigemura (1978) and Cho (1978), the separate boiler cost functions are expressed as

$$
C_{i}=P_{i} \frac{h L_{i}}{\eta_{i}\left(L_{i}\right)}
$$

Received 12 October 1982.

+ Kongsberg Våpenfabrikk A/S, N-3600 Kongsberg.

‡A/S Computas, N-7000 Trondheim. 
where

$h$ enthalphy of steam

$P_{i}$ price per joule of fuel on boiler $i$

$\eta_{i}$ boiler efficiency (load dependent)

$L_{i}$ steam load on boiler $i$

Cho (1978) represented the boiler efficiencies by fitting second-order polynomials to observed boiler data.

In this paper quadratic expressions are used for the boiler efficiencies

$$
\eta_{i}=a_{i} L_{i}{ }^{2}+b_{i} L_{i}+c_{i}
$$

The total steam generation cost to be minimized may then be expressed as

$$
C=\sum_{i=1}^{N} P_{i} \frac{h L_{i}}{a_{i} L_{i}^{2}+b_{t} L_{t}+c_{i}}
$$

With specified $L_{T}$ and known parameters in eqn. (6), the $L_{t} s$ minimizing $C$ can be found by a numerical minimization procedure, which in principle determines the loads recursively.

For ease of notation, let

$$
L=\left[L_{i} \ldots L_{N}\right]^{T}
$$

The numerical minimization, repeated here for easy comparison with a sub-optimal procedure later, is done by the algorithm

$$
L(k+1)=L(k)-\alpha_{k} \nabla C
$$

where $\alpha_{k}$ is determined by minimization of $C$ in the direction $\nabla C(L(k))$.

In the following an optimal solution is compared to two other methods for load allocation.

\section{Three methods for boiler load allocation}

Several alogorithms have been proposed for allocation of steam load to a multiple of boilers in a steam generating plant.

In this paper three methods are considered.

1. The most primitive.

An increase or decrease in steam demand is distributed with an equal portion to each of the boilers.

2. An algorithm for optimal steam load allocation.

3. A method presented in Leffler and Shigemura (1978), which uses the gradient of the cost function in a more simple manner than the optimal algorithm. This method will be referred to as the 'sub-optimal' algorithm.

These methods will be described in more detail.

\subsection{Equal distribution}

According to this, a change, $\beta$, in total steam load demand, will be distributed with the same load change to each of the $N$ boilers

$$
\Delta L_{i}=\frac{\beta}{N}
$$


The most positive that can be said about this criteria from a cost optimization point of view, is that it is simple. It does not take into consideration that boiler efficiency is dependent on boiler load. Only when the boilers have equal initial load, and identical efficiency curves, this method will be of any value.

In this paper equal distribution is only used as a reference, to see what actually can be saved by using more sophisticated methods.

\subsection{Optimal boiler load allocation}

The results on optimal boiler load allocation presented in $\S 4$ were found by solving the optimization problem of $\S 2$ by the use of the method of projected gradients and Davidson's cubic interpolation method.

Having constraints allowing the steam load on boiler $i$ to be zero, made us make the program with $L_{i_{\min }}=0$ for each boiler, but with a special algorithm ensuring that no boiler was allowed to work with a load greater than zero but lower than its real minimum capacity. In our example this worked out very satisfactorily, having negligible impact on the optimization results.

Since the load is allocated statically, the method does not take into account transient losses caused by changing load on each single boiler. An optimal solution is therefore also dependent on how often optimization is performed as steam demand varies with time.

\subsection{The sub-optimal load allocation algorithm}

In order to avoid the line-search procedure needed to determine $\alpha_{k}$ in eqn. (8), and to eliminate the need for calculating $\nabla^{2} C$ for each iteration step, a 'sub-optimal' allocation scheme has been proposed (Leffler and Shigemura 1978), where at every change in plant steam demand, the whole load change is allocated to the boiler which is most cost efficient at the present operating point. In order to decide whether a boiler is cost efficient, the derivative of its cost function with respect to boiler load is computed.

$$
\frac{d C_{i}}{d L_{i}}=P_{i} h\left(\frac{1}{\eta\left(L_{i}\right)}-\frac{L_{i}}{\eta\left(L_{i}\right)^{2}} \frac{d \eta\left(L_{i}\right)}{d L_{i}}\right)
$$

This is known as the gradient of the cost function.

If $\beta$ is positive (load increase), the change is allocated to the boiler with the, at the moment, lowest gradient value. value.

If $\beta$ is negative (load decrease), the change is allocated to the boiler with highest

In cases where the total load change cannot be allocated to the boiler without reaching its upper or lower production limits, the remaining part will be allocated to the next most effective boiler.

The gradient of the total cost function,

$$
\frac{d C}{d L}=\left(\frac{d C_{i}}{d L_{i}} \ldots \frac{d C_{N}}{d L_{N}}\right)^{T}
$$

would be the direction of steepest decent in a search for minimum overall cost, and Aral et al. (1979) argue that this 'sub-optimal' method is approaching optimum as load changes with time.

The authors concede that this solution, which is a step along one axis in the $N$-1-dimensional space, converges slower than a step in the gradient direction, which 
would imply allocating the load change to more than one boiler. It is obvious that the one-boiler load-change scheme also converges slower than a numerical optimization procedure, which will bring the load distribution to the optimal configuration after each change in plant steam demand.

\subsection{Convergence of the 'sub-optimal' algorithm}

It can be shown that this simplified allocation algorithm converges under certain conditions to what is termed 'ideal participation', i.e. the rates of individual boiler loads to the range of the boilers is constant. The conditions stated in (Aral et al. 1979) are rather restrictive: $\beta$ is small compared to the range of the boilers, and the changes in plant steam demand are fluctuations of $\pm \beta$ about an operating point.

It is also claimed that due to varying steam demands, the simplified allocation scheme results in a loading distribution which minimizes the total generating costs, i.e. minimizes $C$ of eqn. (1). In fact, the steam demand changes must satisfy several restrictions for this to be true. In the following analysis, conditions for such convergence are determined. The sub-optimal recursive procedure is

$$
L(k+1)=L(k)+\beta \cdot g(k)
$$

where $\beta$ is the change in steam demand at instant $k+1$ and

$$
g(k)=\left[\left.0 \ldots \frac{d C_{i}}{d L_{i}}\right|_{L_{i}(k)} 0 \ldots 0\right]^{T}
$$

where boiler $i$ have the highest value of $\beta d C_{i} / d L_{i}$ at $L_{i}(k)$.

In order to study the convergence of eqn. (12), the gradient of the minimization criterion is expanded in a Taylor series around the optimal loal distribution $L_{0}$ :

if $L-L_{0}$ is small.

$$
\begin{aligned}
\nabla C(L) & =\nabla C\left(L_{0}\right)+\nabla^{2} C\left(L_{0}\right)\left(L-L_{0}\right)+\ldots \\
& \approx \nabla^{2} C\left(L_{0}\right)\left(L-L_{0}\right)
\end{aligned}
$$

Noticing that

$$
g(k)=H(k) \nabla C\left(L_{k}\right)
$$

where $H(k)$ has only one non-zero element which is 1 in the $(i, i)$ position, we may write

$$
\left.\begin{array}{l}
L(k+1)-L_{0}=L(k)-L_{0}+\beta H(k) \nabla^{2} C\left(L_{0}\right)\left(L(k)-L_{0}\right) \\
\left.\left.L(k+1)-L_{0}=\left[I-\beta H(k) \nabla^{2} C\left(L_{0}\right)\right]\right) L(k)-L_{0}\right)
\end{array}\right\}
$$

The convergence of the sub-optimal algorithm may be determined using eqn. (16). Equation (16) is also written in this form to allow easy comparison with a recursive algorithm for solution of the optimization problem, eqn. (8).

For the sub-optimal algorithm to converge to the true minimum $L_{0}$, two conditions must be met

(i) $\left|I-\beta H(k) \nabla^{2} C\left(L_{0}\right)\right|<1$

(ii) The sequence $H(k) \nabla^{2} C\left(L_{0}\right), k=1, \ldots$ must span the whole optimization space.

Using what we have termed the sub-optimal method, the step size $\beta$ and the step direction $H(k) \nabla^{2} C\left(L_{0}\right)$ is given by plant steam demand and boiler operating conditions, and there is no guarantee that conditions (i) and (ii) will be satisfied during normal plant operation. This is in contrast to the recursive optimal algorithm 
(eqn. (8)), where the step size $\alpha$ is determined by one-dimensional minimization, and the step direction is also a rational choice, e.g. steepest decent.

\subsection{Comparison of the optimal and sub-optimal algorithm}

There is a fair chance, however, that the sub-optimal algorithm converges to a boiler load vector $L$ within a small distance from the optimum when load changes are frequent and small. On the other hand, the loading pattern may be significantly different from the optimal one in periods following large changes in plant steam demand.

Another important factor in the comparison of the sub-optimal and optimal algorithms is the cost function's oscillations around the minimum value, due to the externally given step size. While the step size and number of iterations using the optimal algorithm are determined from a specification of an acceptable accuracy in determining $C\left(L_{0}\right)$, the suboptimal algorithm has to accept the $\beta$ the plant requires. Due to this fact a limit cycle in $C$ will occur even in the case of $\beta$ being small oscillations around an operating point. This may give a significantly larger value of cost integrated over time than what can be achieved using the optimal algorithm.

Because the sub-optimal algorithm uses the plant steam demand changes as excitations, in general a number of such changes must take place before the load allocation can be expected to be in the neighbourhood of the optimal load vector, supposing conditions (i) and (ii) are satisfied.

Depending on the frequency of the steam demand changes, this transient period may also represent significant economic losses, compared to the algorithm which determines the optimal loading pattern after change in steam demand.

The advantage usually claimed for the sub-optimal algorithm is its simplicity, and that it requires less programming and computing effort. Considering the low cost and computional power of the microcomputers of today, we do not see this as a deciding factor.

The fact that the sub-optimal algorithm allocates the change in load to only one boiler (in general) may also be considered an advantage, but it has to be weighted against the possible cost of the large changes in load the boilers may be subject to (e.g. more frequent shut-down and start-up of boilers).

\section{A four boiler example}

To evaluate and compare the methods mentioned above, a model of a steam generating plant with four boilers and a total capacity at $181600 \mathrm{~kg} / \mathrm{h}[400000 \mathrm{lb} / \mathrm{h}]$ was studied. The fuel is oil, and the boiler capacities are as follows:

$$
\begin{array}{ccc}
\text { Boiler } & 1 & 54480 \mathrm{~kg} / \mathrm{h}[120000 \mathrm{lb} / \mathrm{h}] \\
\# & 2 & 36320 \mathrm{~kg} / \mathrm{h}[80000 \mathrm{lb} / \mathrm{h}] \\
\# & 3 & 36320 \mathrm{~kg} / \mathrm{h} \\
\# & 4 & 54480 \mathrm{~kg} / \mathrm{h}
\end{array}
$$

The minimum capacities were said to be $10 \%$ of maximum load capacity. Boiler efficiencies are given by eqn. (5), with parameters given in Table 1 .

To get an impression of how steam demand changes, we used registrations from a Norwegian paper-mill. This installation has a lower maximum capacity than in our model, but the variations should be quite realistic. 


\begin{tabular}{cccc}
\hline & \multicolumn{3}{c}{ Efficiency curve parameters } \\
\cline { 2 - 4 } Boiler no. & $a_{\boldsymbol{i}}$ & \multicolumn{1}{c}{$b_{\boldsymbol{i}}$} & $c_{\boldsymbol{i}}$ \\
\hline 1 & $-1 \cdot 755 \cdot 10^{-11}$ & $3 \cdot 817 \cdot 10^{-7}$ & $0 \cdot 86$ \\
2 & $-1 \cdot 774 \cdot 10^{-11}$ & $2 \cdot 023 \cdot 10^{-7}$ & $0 \cdot 85$ \\
3 & $-3 \cdot 949 \cdot 10^{-11}$ & $2 \cdot 01 \cdot 10^{-6}$ & $0 \cdot 85$ \\
4 & $-2 \cdot 911 \cdot 10^{-11}$ & $2 \cdot 093 \cdot 10^{-6}$ & $0 \cdot 84$ \\
\hline
\end{tabular}

$(\operatorname{dim}[L]=\mathrm{kg} / \mathrm{h})$

Table 1.

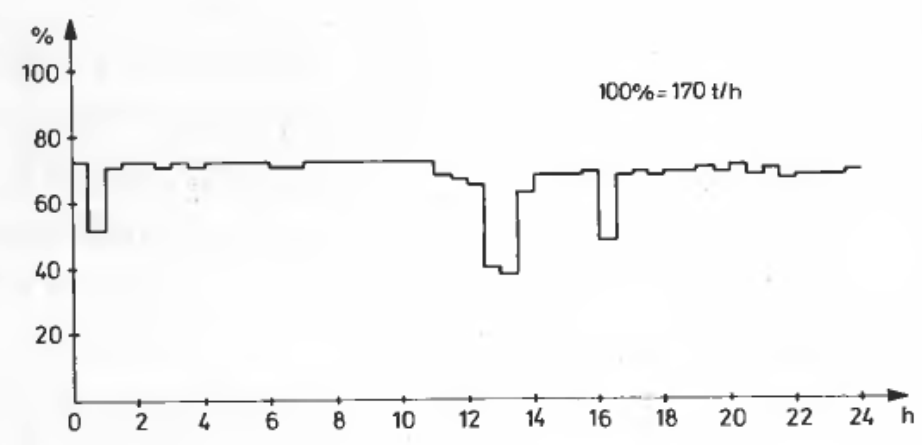

Figure 1. Steam demand, low activity period.

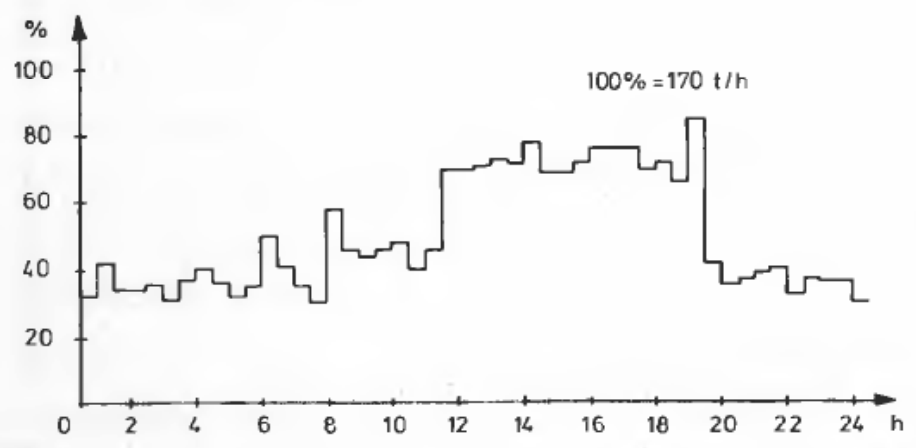

Figure 2. Steam demand, high activity period.

By sampling these registrations every half hour, we concentrated on two periods of $24 \mathrm{~h}$ each. One with relatively small and few changes, and the other with larger and more frequent variations. Here the periods are called the low- and high-activity periods, respectively. The steam load to be allocated is shown in Figs. 1 and 2.

This four-boiler example was used to compare the equal distribution, optimal and sub-optimal load allocation schemes. Results from there cases are given here:

(a) Equal allocation versus optimal allocation in a $120 \mathrm{~h}$ period

Optimization gives a cost reduction of approx. $0.8 \%$. This gives an impression of how much you lose by not using any kind of optimization method. 

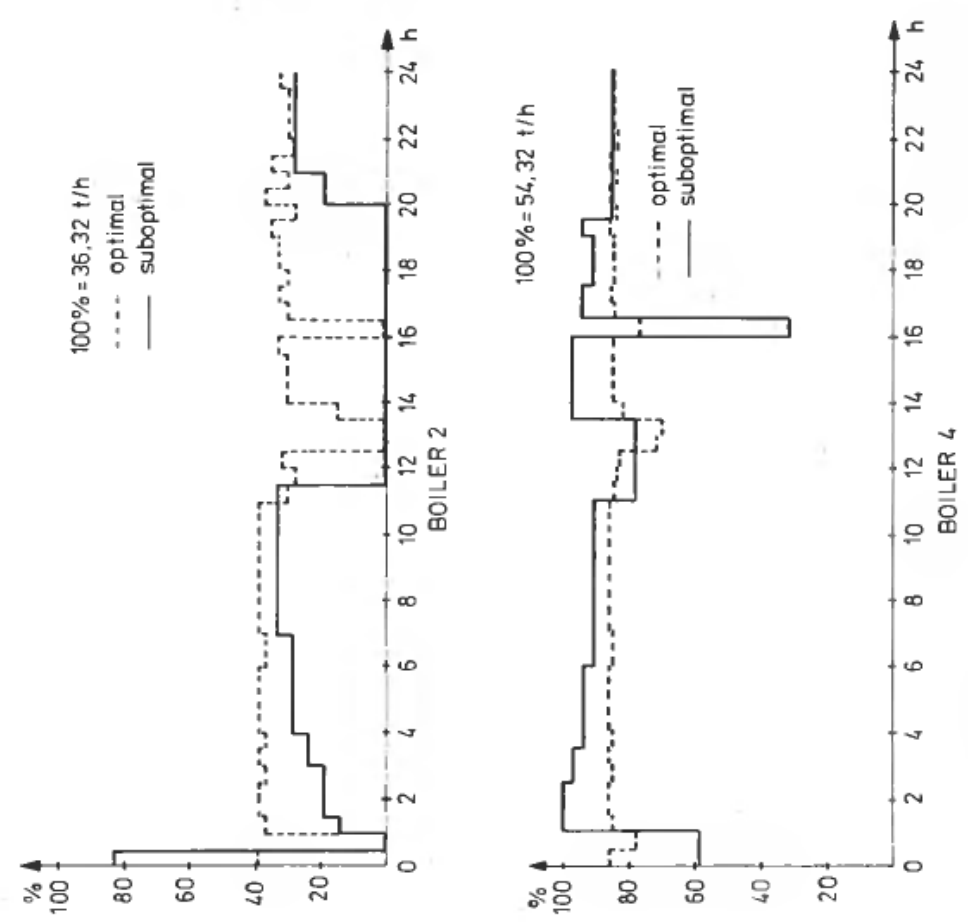

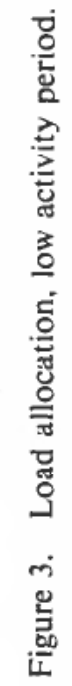
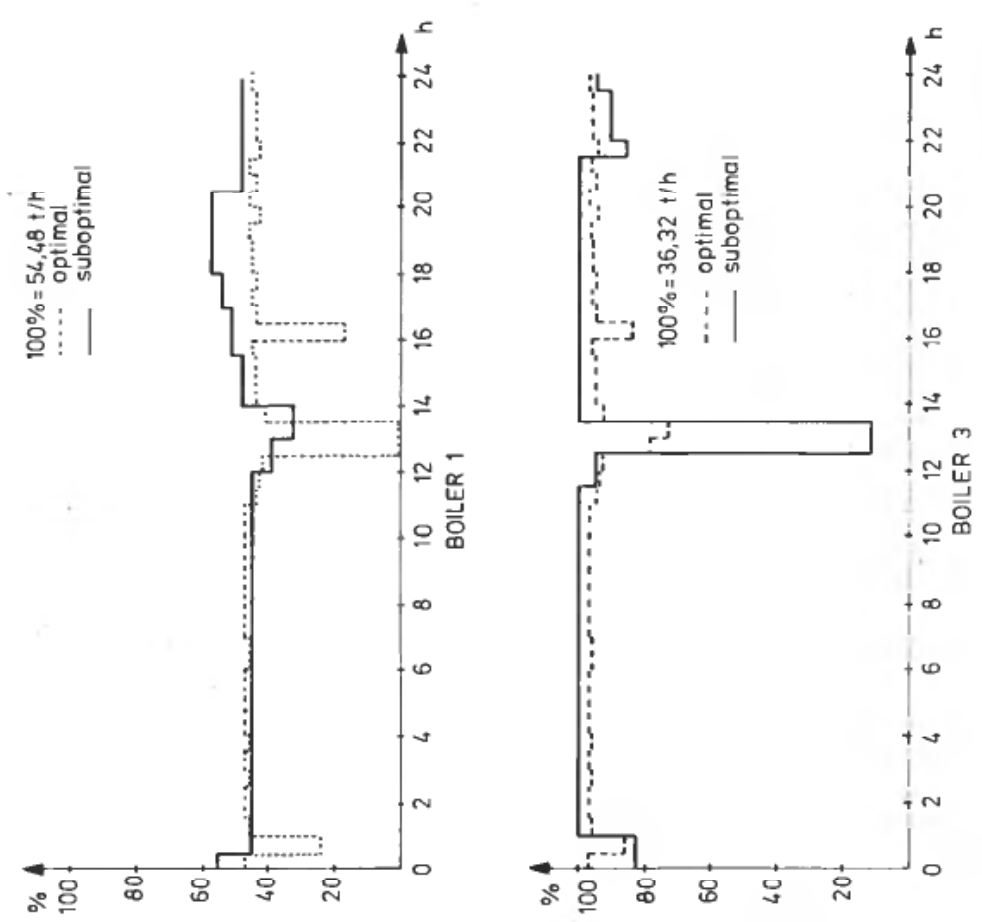
(b) Optimal allocation versus sub-optimal in the low activity period

Since the suboptimal method is dependent on the load allocation when the algorithm starts to work, it was assumed an initial total load of $120000 \mathrm{~kg} / \mathrm{h}$ with $30000 \mathrm{~kg} / \mathrm{h}$ on each boiler.

Optimal allocation gave a cost $0.11 \%$ lower than the sub-optimal method. Compared to an allocation with equal portions, optimization would mean a cost reduction of $0.73 \%$.

The steam loads on each boiler using sub-optimal and optimal methods are illustrated in Fig. 3.

(c) Optimal allocation versus sub-optimal in the high activity period Initial load: $100000 \mathrm{~kg} / \mathrm{h}$ with $25000 \mathrm{~kg} / \mathrm{h}$ on each boiler.

Here the optimization method gave a cost $0.29 \%$ lower than the sub-optimal method.

Compared to the method with equal load allocation, optimization gives a cost that is $1 \cdot 1 \%$ lower.

Figure 4 illustrates the boiler loads in this period, using sub-optimal and optimal methods.

(d) The above results are summarized in Table 2.

\begin{tabular}{lcc}
\hline & $\begin{array}{c}\text { Optimal versus } \\
\text { suboptimal }\end{array}$ & $\begin{array}{c}\text { Optimal versus } \\
\text { equal }\end{array}$ \\
\hline 120 h per. & - & $0.8 \%$ \\
Low activity & $0.11 \%$ & $0.73 \%$ \\
High activity & $0.29 \%$ & $1 \cdot 1 \%$ \\
\hline
\end{tabular}

Table 2. Cost reduction using optimization.

\section{Comments}

Since the differences are so small, and the results presented above are results of static computations, it is difficult to draw general conclusions about the methods. However, in our example some characteristics of the methods can be observed.

(a) The optimal algorithm

From Table 1 and eqn. (5) it can be seen that for most loads, boilers 3 and 4 are more efficient than the two others. From this it was expected that in periods with low total steam demand, boilers 1 and 2 would get very little load. This is confirmed by our results. In fact, in the high activity period with low steam demand in the beginning, boilers 1 and 2 do not get any load at antil after about $5 \cdot 5$ and 11 hours, respectively.

Further we see that the optimal allocation algorithm changes the load of every boiler almost every time the total steam demand changes. These individual load 

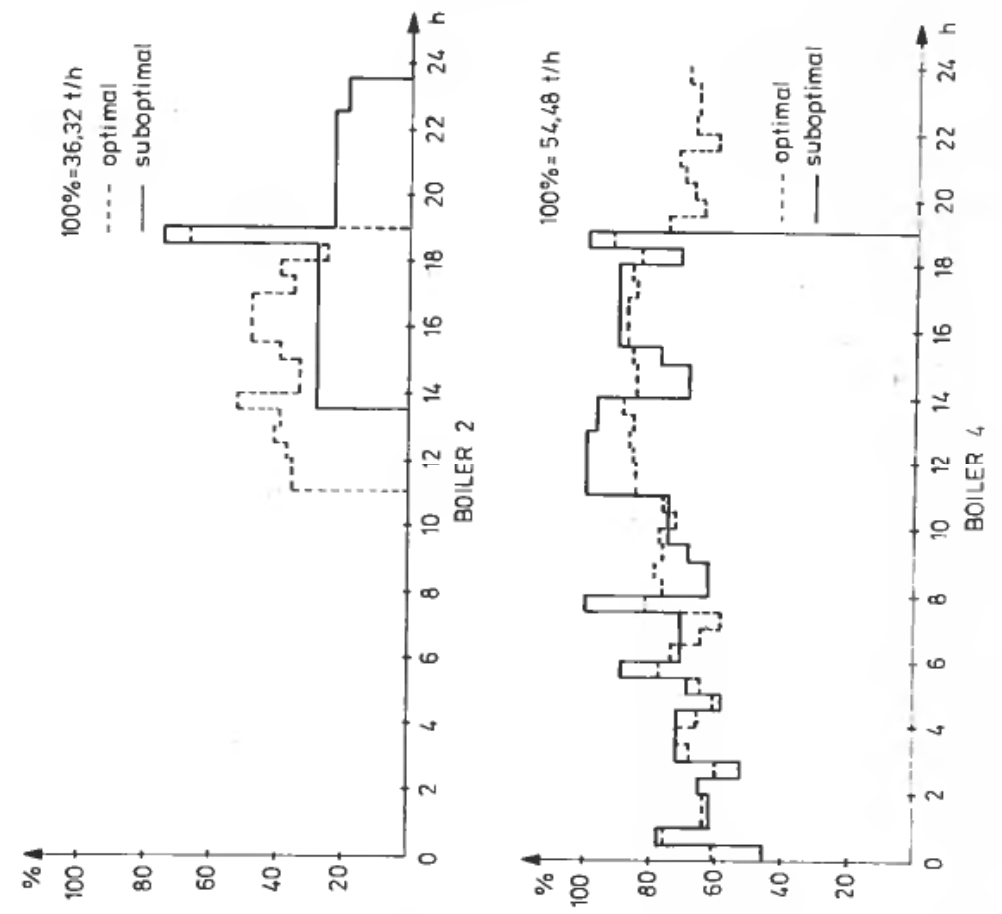

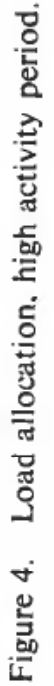

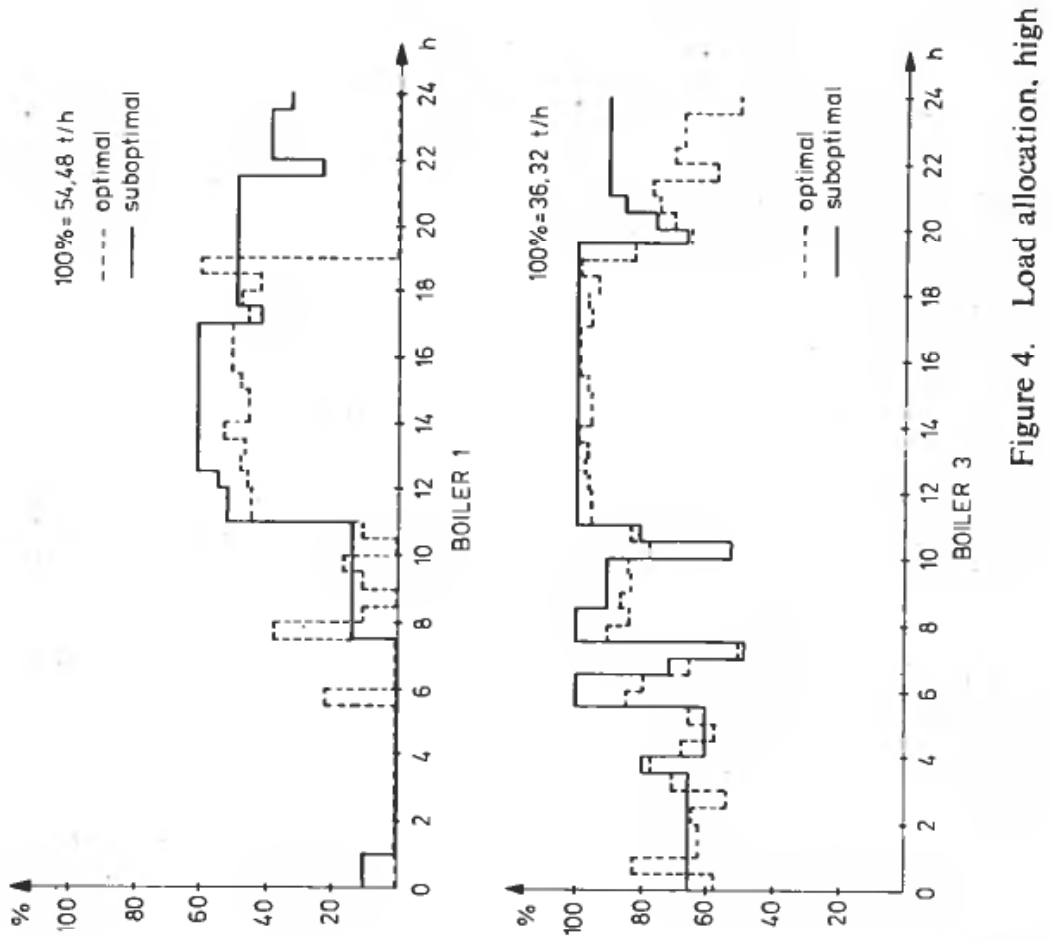


changes are relatively small most of the time, but since they are so frequent, they may be significant in a real-life implementation.

(b) The sub-optimal algorithm

As this method allocates the total change in steam demand to the most cost efficient boiler, the individual load changes are not so frequent, although they may be large.

Under certain conditions, this turns out to be rather unfavourable, as can be seen from Fig. 4. At the end of the high activity period, boiler 4 is turned off, while it optimally should have approximately $70 \%$ load.

The results reported here are also consistent with the analysis and comments of $\S 3$, in that the sub-optimal algorithm gives a loading pattern close to the optimal only when the total demand is subject to small variations, like in the first half of the low activity period. When a large change in steam demand occurs, the sub-optimal algorithm tends to deviate from the minimum cost solution.

When the steam demand varies slowly around an average value, the two methods give almost the same allocation pattern. If the variations are like the high activity period, the sub-optimal algorithm cannot compete with optimal allocation in our example.

\section{Conclusions}

Two methods (termed optimal and sub-optimal) of on-line allocation of boiler loads have been compared theoretically and by an application example. Questions concerning the convergence of the 'sub-optimal' allocation algorithm [2] have been discussed and illustrated by examples using the plant demand of a large Norwegian paper mill. While both methods gave cost reductions compared to equal distribution of the load, the optimal load allocation algorithm gave an added reduction in cost of $0.1 \%$ to $0.3 \%$ relative to the cost of using the sub-optimal algorithm, and of 0.7 to $1 \cdot 1 \%$ relative to equal distribution. Due to high energy costs, even small percentages may represent a substantial sum.

With the microcomputers of today, the optimal allocation algorithm is easily implemented.

\section{REFERENCES}

ANDREASSEN, Ø. (1980). Optimal allocation of steam production between several boilers. M.Sc. Thesis, Division of Engineering Cybernetics, The Norwegian Institute of Technology. (In Norwegian.)

Aral, G., VAN WINKLE, R., Al-ShaikH, A. (1979). The power boiler and steam distribution system of a paper mill - a prime example of energy optimization. 5th Ann. Control Conf., Purdue. E. J. Kompas, T. J. Williams (eds.). Techn. publ. Co., pp. 97-114.

Сно, С. Н. (1978). Optimum boiler load allocation. Instrumentation Teclinology, 25, pp. 55-58. LeFFler, N., and SHIGENURA, M. (1978). Optimal steam allocation of a set of multifuel power boilers. in A. Niemi (ed); A link between science and applications of automatic control, Pergamon Press, pp. 89-96. 